\title{
Saúde do adolescente em Medicina Geral e Familiar
}

Cristina Ribeiro,* Inês Rosendo*

«Levada pela sua curiosidade a seguir o coelho, Alice vê-se a cair até ao centro da terra povoado de todas as suas fantasias».

Adaptado In Alice no país das maravilhas Lewis Carroll

(Autor da história Alice no País das Maravilhasfoi Lewis Carroll. Bem... na verdade foi Charles Lutwidge Dodgson. Era inglês e nasceu em $1832 \mathrm{e}$ foi o primeiro filho de uma família com onze crianças...)

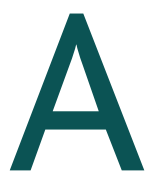
adolescência (do latim adolescere que significa crescer) é definida como o período de vida situado entre a infância e a idade adulta, começando na puberdade, com o aparecimento dos caracteres sexuais secundários, e terminando com o fim do crescimento, ${ }^{1}$ pressupondo o atingimento da maturidade psicofísica. Com características dependentes de tantas variações individuais e sócio-culturais, compreende-se ser difícil estabelecer idades limite para definir este período: a OMS considera entre os 10 e os 19 anos inclusivé, ${ }^{2}$ a Associação Internacional para a Saúde dos Adolescentes (IAAH) entre os $10 \mathrm{e}$ os 24 anos, ${ }^{3}$ e a DGS entre os 10 e 18 anos. ${ }^{4}$ Os estádios do desenvolvimento humano de Erikson consideram um adolescente, na fase de agregação das características da identidade para atingir a idade adulta, dos 13 aos 19 anos. $^{5}$

A adolescência é caracterizada por mudanças físicas, cognitivas, psicológicas e sociais. O próprio ciclo vital familiar se modifica quando um filho entra na adolescência pela sua busca de maior autonomia e socialização. ${ }^{6,7}$ Entre as dificuldades e riscos com que se deparam os adolescentes destacam-se o consumo de álcool, tabaco e outras drogas, as primeiras experiências sexuais, a associação em grupos, os distúrbios do com-

*Editora da RPCG. portamento alimentar e os possíveis comportamentos socialmente agressivos.

Há poucos estudos sobre o efeito da vigilância e intervenção em adolescentes pela parte dos médicos de família. Há alguns que mostram que podem conseguir influenciar positivamente o seu comportamento relacionado com saúde (tabaco, álcool, nutrição e actividade sexual). ${ }^{8,9,10}$ A DGS recomenda consultas de vigilância aos 11-13, 15 e 18 anos. ${ }^{4}$

De acordo com os estudos sobre as suas expectativas, ${ }^{11,12,13,14}$ sabe-se que as consultas a adolescentes devem ter particularidades próprias, como a facilitação do acesso (gratuita, desburocratizada e podendo ser atendidos), a garantia de confidencialidade, o carácter despreconceituoso e desprovido de julgamentos, o menor tempo de espera possível e envolvendo activamente. ${ }^{4,15,16}$

Os objectivos desta consulta serão promover a auto-estima do adolescente e a sua progressiva responsabilização pelas escolhas relativas à saúde, prevenindo situações disruptivas ou de risco acrescido. Assim, o Médico de Família pode fazer o acompanhamento de todas as transformações que acontecem neste período construindo um espaço e tempo ideal para esclarecimento de dúvidas, partilha de experiências, prevenção e detecção precoce de disfunções/alterações, assim como acompanhamento dos processos e estratégias de resolução das diferentes pequenas grandes crises que caracterizam esta fase. É também um momento privilegiado para a promoção e adopção de estilos de vida saudáveis (em continuidade com o trabalho em idades mais precoces) que, uma vez assimilados nesta idade, na maioria dos casos se manterão na vida adulta. . $^{4,17,18}$

O papel de prevenção e intervenção da equipa de cuidados de saúde primários pode estender-se mesmo fora do Centro de Saúde, planeando intervenções com

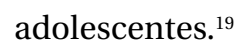

Existe cada vez mais a percepção de que é importante formação nesta área em Medicina Geral e Fami- 
liar. ${ }^{20,21}$ Em Portugal, no programa de formação em Medicina Geral e Familiar, existem objectivos especificamente dirigidos à formação em saúde do adolescente, enquadrado no estágio de pediatria, ${ }^{22}$ mas na prática muitos não obtêm formação nesta área.

Todas as dimensões anteriormente referidas são essenciais para que o papel do médico de família em Cuidados de Saúde Primários possa contribuir de um modo integrado para uma prática que proporcione junto do adolescente a intervenção mais apropriada.

Durante esta etapa do ciclo de vida, com a puberdade, o organismo sofre várias modificações que afectam, sucessivamente, todos os aspectos da vida biológica, psicológica e social: 1) alterações corporais, com emergência dos caracteres sexuais secundários e vivência de impulsos e tensões desconhecidos; 2) emergência da sexualidade, com conquista de um novo tipo de relação com o outro e do espaço individual, implicando modificações na relação com os pais, maior preocupação consigo próprio, vivência de emoções intensas e de inquietações a nível da identidade; 3 ) ao nível do pensamento e da forma de abordar o real (alterações cognitivas), alargamento da perspectiva temporal, maior espírito crítico, maior capacidade de insight, maior capacidade de decisão e de autonomia moral e maior capacidade de utilizar a linguagem; 4) a nível social (com a emancipação da tutela parental e o estabelecimento de novas relações entre os pares), procura de outros modelos de identificação, organização de uma nova rede relacional e apropriação crítica dos valores sociais e familiares.

Todas estas dimensões constituem pressupostos fundamentais para que neste Dossier pudéssemos contar com algumas temáticas necessárias de serem sistematizadas em suportes úteis na prática clínica do Médico de Família. Assim Cristina Freitas, Helena Sofia Sousa e Helena Fonseca, em dois artigos, sistematizam o que de primordial deve ser considerado no Boletim de Saúde Infantil e Juvenil - o exame global de saúde dos 11 aos 13 anos. Os autores apresentam vários aspectos que devem ser contemplados pelo profissional de saúde quando da observação de um adolescente desta idade. No parte I são abordados os aspectos a englobar na anamnese através da utilização do acrónimo HEEADSSSSS, com o objectivo de avaliar os contextos de vida do adolescente e perceber qual o seu desen- volvimento cognitivo e psicossocial. Na parte II são abordadas as alterações mais frequentemente encontradas no exame objectivo e apresentadas várias medidas preventivas e as atitudes terapêuticas recomendadas. Finalmente, são feitas algumas sugestões tendo como objectivo uma melhoria no atendimento e avaliação global dos adolescentes nesta faixa etária.

O objectivo do artigo sobre Perturbações do Comportamento Alimentar na Adolescência é o de examinar como algumas particularidades, nomeadamente relacionadas com a epidemiologia, o diagnóstico, as complicações médicas e nutricionais, as questões psicológicas, o tratamento e o prognóstico dos adolescentes com uma Perturbação do Comportamento Alimentar diferem dos adultos, sobretudo no que respeita à prevenção e às questões ligadas com o desenvolvimento que caracterizam este período. Por último, e não menos importante, Isabel Brito aborda conteúdos que têm a ver com as perturbações da ansiedade e depressivas na adolescência. Estas perturbações provocam situações problemáticas na família, na escola e socialmente. São destacadas algumas competência do médico de família, que segue estes doentes ao longo do seu desenvolvimento, no sentido de os avaliar, tratar e referenciar. A confiança que a família e o adolescente depositam neste profissional de saúde permite-lhe controlar a evolução e prevenir situações de risco do jovem com o suporte familiar.

\section{REFERÊNCIAS BIBLIOGRÁFICAS}

1. Manuila L, Manuila A, Lewalle P, Nicoulin M. Dicionário Médico. $3^{\mathrm{a}}$ ed. Lisboa: Climepsi; 2004.

2. WHO. Adolescent Friendly Health Services: an agenda for change. Geneva: World Health Organization; 2002.

3. International Association for Adolescent Health. Disponível em: http://www.iaah.org/files/iaah.htm [acedido em 01/03/2011].

4. Direcção-Geral da Saúde, Divisão de Saúde Materna, Infantil e dos Adolescentes. Saúde Infantil e Juvenil: Programa-tipo de actuação. $2^{\text {a }}$ ed. Lisboa: DGS; 2005.

5. Wilder EJ. The theoretical basis for the life model. The Complete Guide to Living With Men, 2003. Disponível em: http://www.lifemodel.org [acedido em 01/03/2011].

6. De la Revilla L. La atención longitudinal: el ciclo vital familiar. Conceptos e instrumentos de la Atención Familiar. Barcelona: Doyma; 1994. p. 37-42.

7. Marcos B. La adolescencia en el contexto familiar. Manual de Atención Familiar (II): bases para la práctica familiar en consulta, Vol. II. Granada: Adhara; 1996. p. 659-94.

8. Klein D. Adolescents' health. Does having a family physician make a dif- 
ference? Can Fam Physician 2003 Aug; 49 (8): 1000-2.

9. Walker ZA, Townsend J. The role of general practice in promoting teenage health: a review of the literature. Fam Pract 1999 Apr; 16 (2): 164-72.

10. Walker ZA, Oakley LL, Townsend JL. Evaluating the impact of primary care consultations on teenage lifestyle: a pilot study. Methods Inf Med 2000 Aug; 39 (3): 260-6.

11. Gleeson CR, Robinson MB, Neal RD. A review of teenager's perceived needs and access to primary health care: implications for health services. Prim Health Care Res Dev 2002 Jul; 3 (3):184-93.

12. Oandasan I, Malik R. What do adolescent girls experience when they visit family practitioners? Can Fam Physician 1998 Nov; 44: 2413-20.

13. Ginsburg KR, Slap GB, Cnaan A, Forke CM, Balsley CM, Rouselle DM. Adolescents' perceptions of factors affecting their decisions to seek health care. JAMA 1995 Jun 28; 273 (24): 1913-8.

14. Klein D, Wild TC, Cave A. Understanding why adolescents decide to visit family physicians - qualitative study. Can Fam Physician 2005 Dec; 51:1660-1.

15. Klein JD, Slap GB, Elster AB, Schonberg SK. Access to health care for adolescents: a position paper for the Society of Adolescent Medicine. J Adolesc Health 1992 Mar; 13 (2): 162-70.

16. WHO. Adolescent friendly health services: making it happen. Geneva: World Health Organization; 2005.

17. Direcção-Geral da Saúde. Saúde Juvenil - Programa Nacional de Saúde dos Jovens - Proposta. Lisboa: Direcção-Geral da Saúde, 2005. Disponível em: http://www.acs.min-saude.pt/wp-content/up loads/ 2007/ 12/programanacionaldesaudejovens.pdf [acedido em 01/03/20111].

18. Tavares HB, Fonseca H. Avaliação dos recursos de atendimento ao adolescente nas unidades hospitalares portuguesas. Acta Pediatr Port 2009 Jul-Ago; 40 (4): 154-9.

19. Cordeiro RA. O planeamento de um programa de intervenção com adolescentes. Rev Port Clin Geral 2007 Nov-Dez; 23 (6): 709-11.

20. Blum RW, Bearinger LH. Knowledge and attitudes of health professionals toward adolescent health care. J Adolesc Health Care 1990 Jul;11(4): 289-94.

21. Graves CE, Bridge MD, Nyhuis AW. Residents' perception of their skill levels in the clinical management of adolescent health problems. J Adolesc Health Care 1987 Sep; 8 (5): 413-8.

22. Portaria $n^{\circ} 300 / 2009$, de 24 de Março. Diário da República, 1.a série N 58. 24 de Março de 2009.

\section{CONFLITOS DE INTERESSES}

As autoras declaram não possuir conflitos de interesse.

\section{ENDEREÇO PARA CORRESPONDÊNCIA}

cristina.mpr@sapo.pt

inesrcs@rpcg.apmcg.pt 